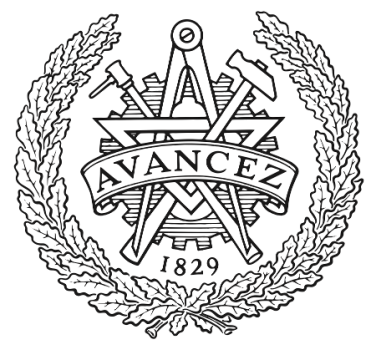

CHALMERS

UNIVERSITY OF TECHNOLOGY

\title{
Low-Complexity Variable-Length Output Distribution Matching with Periodical Distribution Uniformalization
}

Downloaded from: https://research.chalmers.se, 2023-04-26 13:22 UTC

Citation for the original published paper (version of record):

Yoshida, T., Karlsson, M., Agrell, E. (2018). Low-Complexity Variable-Length Output Distribution Matching with Periodical Distribution Uniformalization. 2018 OPTICAL FIBER COMMUNICATIONS CONFERENCE AND EXPOSITION (OFC). http://dx.doi.org/10.1364/OFC.2018.M4E.2

N.B. When citing this work, cite the original published paper. 


\title{
Low-Complexity Variable-Length Output Distribution Matching with Periodical Distribution Uniformalization
}

\author{
Tsuyoshi Yoshida ${ }^{1,2}$, Magnus Karlsson ${ }^{1}$, and Erik Agrell ${ }^{1}$ \\ ${ }^{1}$ Chalmers University of Technology, SE-41296 Gothenburg, Sweden \\ ${ }^{2}$ Mitsubishi Electric Corporation, 5-1-1 Ofuna, Kamakura, Kanagawa, 247-8501 Japan \\ tsuyoshi@chamlers.se,Yoshida.Tsuyoshi@ah.MitsubishiElectric.co.jp
}

\begin{abstract}
Run-length code based distribution matching (DM) for probabilistic shaping is combined with a uniformalizer to realize low-complexity fixed-length DM. The proposed method is $0.4 \mathrm{~dB}$ better than previous low-complexity DM methods.

OCIS codes: (060.4510) Optical Communications; (060.4080) Modulation
\end{abstract}

\section{Introduction}

A number of schemes for probabilistic constellation shaping have been investigated to approach the Shannon capacity. Probabilistic amplitude shaping [1] with arithmetic coding-based constant-composition distribution matching (CCDM) [2] is one of the state-of-the-art techniques in the optical communication field, due to its asymptotically optimal performance and fixed-length to fixed-length (f2f) conversion feature. Turbo coding with many-to-one mapping with iterative demodulation also shows very good performance [3]. On the other hand, both these schemes are complex when implemented in hardware for high-throughput optical communication systems. For the techniques in [1,2], a bit-wise distribution matching (DM) and its superposition will relax the complexity issue somewhat [4-6]. The simplest implementation of bit-wise DM by arithmetic coding, which would be an $m$-out-of- $n$ code [7], requires two multipliers per bit with high-precision arithmetics ( $\geq 12$ bits quantization [7,8]). Significantly less complex shaping techniques can be found in [6,9], but with more limited performance improvements. Thus, it is still desirable to realize DMs with a better tradeoff between performance and complexity.

Arithmetic coding is a well-known entropy code that originally was used for data compression. There are simple arithmetic coding families like [10], but it is not known if they can be applied to an f2f-DM. Recently, stream DM has been proposed [8], which switches several variable-length output codebook tables to realize quasi-f2f-DM without multiplications. However, easier compression techniques than arithmetic coding could be an alternative candidate. In this paper, we extend our previous shaping scheme in [6] by introducing a new bit-wise DM, which uses a mapper based on run-length coding (RL mapper). Regular run-length codes are impractical, since their output length vary with the input data, i.e., they are not f2f. As we will show, however, an f2f-DM can be realized by employing distribution unifomalizers, which makes the number of marks and spaces per unit block exactly the same in each input bit lane to the RL mapper. The proposed DM is bounded in length because of the uniformalization feature, so error propagation due to residual errors after forward error correction (FEC) decoding will be prevented.

\section{The proposed DM technique and uniformalization}

The DM in this paper is an extended version of the previous work in [6], which was based on a bit-level DM [4,5] and low-complexity bit-wise DMs [6]. In this paper, we introduce a bit-wise DM with improved performance relative to [6]. To save space, here we explain only our new bit-wise DM in detail. It is based on run-length decompression, which can realize $\mathrm{f} 2 \mathrm{f}$ conversion with limited-length buffering by using distribution uniformalizers (UFLs). The schematic of the bit-wise DM is shown in Fig. 1. Binary uniform (at infinite length) input bits are demultiplexed to $K$ parallel bit lanes. Each UFL makes the mark ratio exactly $1 / 2$ at the output (at a finite length), which will be explained later. The RL mapper converts a fixed number $K$ of parallel input bits to variable-length output words. An example of RL mapping is shown in Tab. 1, where the number of input bit lanes $K$ is 3 . The

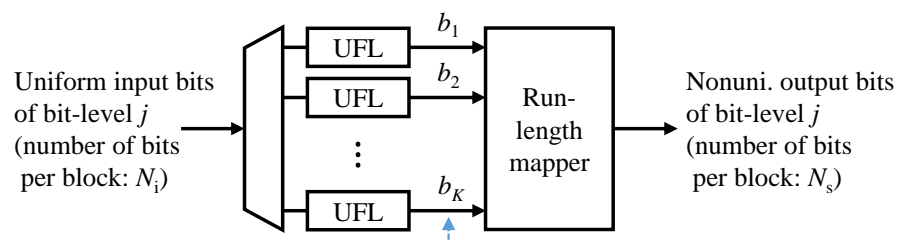

Mark ratio of each UFL output equals exactly $1 / 2$ per block

Tab. 1. An example of a RL mapper for $K=3$.

\begin{tabular}{|c|c|l|c|}
\hline $\begin{array}{c}\text { Input bits } \\
b_{1} b_{2} b_{3}\end{array}$ & $\begin{array}{c}\text { Input } \\
\text { symbol }\end{array}$ & $\begin{array}{c}\text { Output } \\
\text { bits }\end{array}$ & $\begin{array}{c}\text { Output } \\
\text { length }\end{array}$ \\
\hline 000 & 0 & 1 & 1 \\
\hline 001 & 1 & 01 & 2 \\
\hline$\ldots$ & $\ldots$ & $\ldots$ & $\ldots$ \\
\hline 110 & 6 & 0000001 & 7 \\
\hline 111 & 7 & 00000000 & 8 \\
\hline
\end{tabular}

Fig. 1. Schematic of the proposed bit-wise DM. UFL: uniformalizer. 
number of consecutive spaces in the output word is given by the input word interpreted as an integer, so the output word length varies from 1 to $2^{K}$. All cases can be distinguished by just reading from the head of the received bits. The input bits to the RL mapper in each bit lane is completely uniform during a block. It can be shown that this makes the average output block length, per $K$ input bits, exactly $\left(1+2^{K}\right) / 2$, thus ensuring an $\mathrm{f} 2 \mathrm{f}$ mapping of bits.

The rate loss (output entropy minus information rate) as a function of output entropy is shown in Fig. 2 . For comparison, the schemes of [6] are also shown. The rate loss of the permutation mapper and the bit scramble mapper are far from the ideal case, especially at entropies in the range $0.3-0.8$, because of the simplicity and limited output block length $N_{\mathrm{s}} \leq$

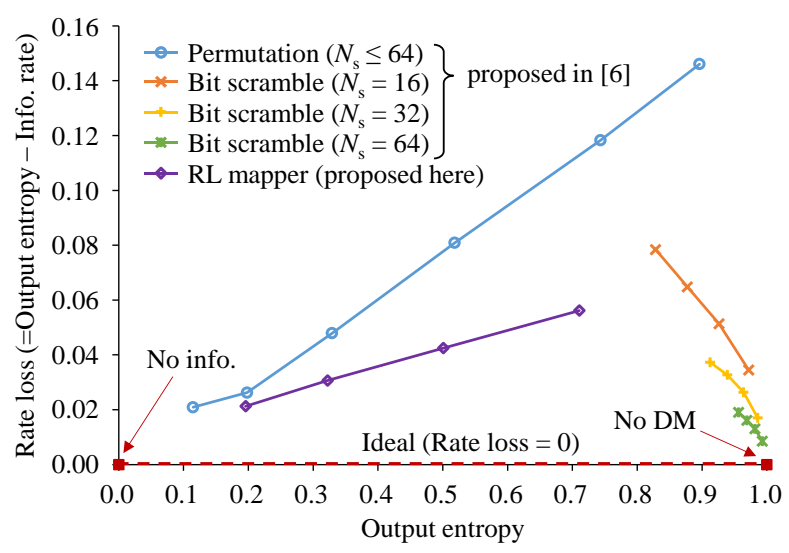

Fig. 2. Rate loss vs. output entropy of low-complexity bit-wise f2f-DMs. 64, limited number of marks (2) for the permutation mapper, and limited number of bit scramble patterns (8). However, the RL mapper is much closer to the ideal performance. For high entropies, $0.8-1$, bit scramble mappers are preferred.

Fig. 3 shows how the proposed UFL works. For given integers $K, L$, and $M$, a block of $N_{\mathrm{i}}=K[(L-1)(M-1)+1]$ input bits is first demultiplexed into $K$ parallell lanes. In each lane, the bits are further divided into $M-1$ subblocks of $L-1$ bits each, except the first that has $L$ bits. For the first subblock, we just count the number of marks. For the remaining subblocks, we either flip all bits in the subblock or not. The flip information is carried by 1 bit per subblock. By doing so, the total difference between the number of marks and spaces will be bounded within $\pm L$ bits. An $M^{\text {th }}$ subblock is therefore appended, consisting of a mark-count sequence to clean up the residual mark-space difference, making it zero over the entire block of $L M$ output bits. Finally, the output bits are RL-mapped as described above and multiplexed into a single output block of $N_{\mathrm{s}}=L \times M \times\left(1+2^{K}\right) / 2$ bits. The error propagation in the distribution dematching will thus be limited to $N_{\mathrm{s}}$ bits even if the FEC decoder fails to correct some errors.

Fig. 4 shows 10 examples of the offset of the output block length after the RL mapper without (a) and with (b) distribution uniformalization, where $K=6, L=128$, and $M=16$. The offset after subblock $i$ is defined as the cumulative output block length minus the target length of $L \times i \times\left(1+2^{K}\right) / 2$. The offsets diverge significantly without uniformalization in Fig. 4(a), but settles to zero with it in Fig. 4(b).

\section{Parameters of the run-length mappers and performance results}

Tab. 2 summarizes the parameters of the studied run-length mappers with uniformalizers. For simplicity, we define only four RL mappers, realized by look-up tables with $2^{K}$ entries. The parameters were chosen so that $N_{\mathrm{s}}$ is the same in all four cases.

The simulated constellation gain as a function of modulation and shaping spectral efficiency is shown in Fig. 5 for $2^{m}$-ary pulse amplitude modulation, where $m=2,3,4$, or 5 . We chose $m-1$ bit-wise DMs from the four RL mappers in Tab. 2, the twelve bit scramble mappers [6], no DM, and no information (e.g. all bits zero) shown in Fig. 2. The performance of the previous low-complexity DM [6] (permutation mapper and bit scramble mapper in Fig. 2) is also shown for comparison. The constellation gain $G$ of probabilistically shaped signals depends on the FEC code rate $R_{\mathrm{c}}$ [6], so we calculated it at $R_{\mathrm{c}}=1$ and 5/6 in Figs. 5 (a) and (b), resp. There are $(m-1)^{18}$ cases, and the best

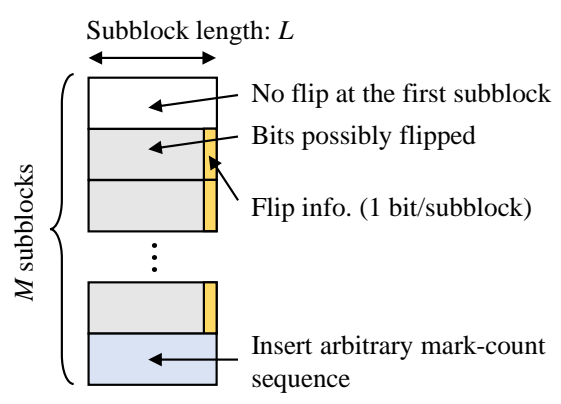

Fig. 3. Bit-wise DM block structure and uniformalization processing
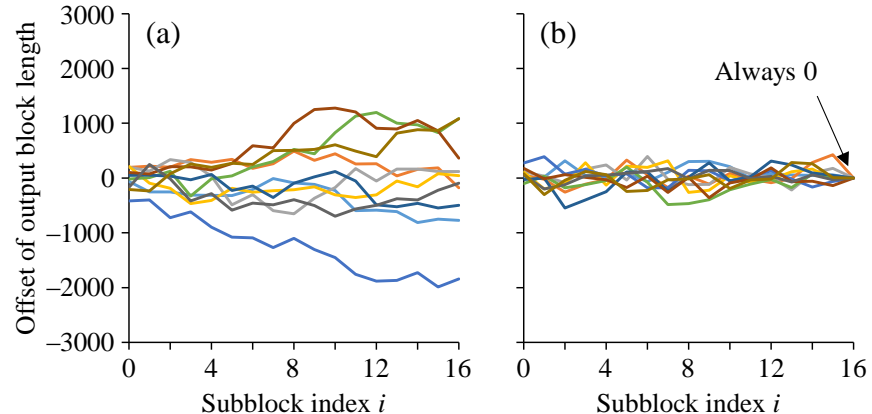

Fig. 4. Offset of the output block length of the RL mapper (a) without and (b) with distribution uniformalizer. 
combination is shown at each (discretized) spectral efficiency. We observed performance dips at 3, 5, 7, and $9 \mathrm{~b} / \mathrm{s} / \mathrm{Hz} / \mathrm{pol}$. for the scheme in [6], but we see no similar dips for the proposed scheme. The maximum improvement over [6] is around $0.5 \mathrm{~dB}$ at such spectral efficiencies even when we take the code rate into account (then performance with

Tab. 2. Parameters of RL mappers with distribution uniformalizers.

\begin{tabular}{|c|c|c|c|c|c|c|c|}
\hline$\#$ & $N_{\mathrm{i}}$ & $L$ & $M$ & $K$ & $N_{\mathrm{s}}$ & $\begin{array}{c}\text { Info. } \\
\text { rate }\end{array}$ & Entropy \\
\hline 1 & 71997 & 143 & 170 & 3 & 109395 & 0.6581 & 0.7107 \\
\hline 2 & 50580 & 110 & 117 & 4 & 109395 & 0.4624 & 0.5008 \\
\hline 3 & 32345 & 78 & 85 & 5 & 109395 & 0.2957 & 0.3223 \\
\hline 4 & 19506 & 51 & 66 & 6 & 109395 & 0.1783 & 0.1958 \\
\hline
\end{tabular}
stronger shaping becomes lower).

We also simulated the required signal-to-noise ratio (SNR) gap from the Shannon capacity on the Gaussian channel. As the information rate, we evaluated the rate $2 R_{\max }-\left(1-R_{\mathrm{c}}\right) 2 m$, where $R_{\max }$ and $m$ are the rate per one dimensions without FEC coding and the number of bits per one dimension, resp. We used the DVB-S2 low-density parity-check soft-decision FEC codes [11] having code rates from 2/3, 3/4, 5/6, and 9/10 with 20 decoding iterations, and an outer hard-decision FEC code [12] with a code rate of 0.9922 . The error-free $\left(10^{-15}\right)$ threshold for the outer FEC code is at a bit-error rate (BER) of $5 \times 10^{-5}$ [12]. The results are shown in Fig. 6 . We chose 6 cases from Fig. 5 for the proposed RL mapping with bit scramble mapping with $2 R_{\max }=2.59,3.32,4.56,4.89,5.32,6.20$, $6.44,6.92,7.22,8.44$, and 9.16 b/s/Hz. For comparison, uniform 4, 8, 16, 32, 64, 128, 256, and 1024-QAM, CCDM [2] based 64-QAM with $N_{\mathrm{s}}=1024$, and the previous low-complexity f2f-DMs [6] are also shown (each of them is degraded around $0.2 \mathrm{~dB}$ compared to [6] due to the evaluation at the post-FEC BER of $10^{-15}$ instead of 10 ${ }^{-3}$ in [6]). Soft-decision FEC code rates for the shaped signals are $>(m-1) / m$. While CCDM shows the best performance at the expense of significant complexity, the proposed DM shows around $0.4 \mathrm{~dB}$ (at around 2, 4, 6, and $8 \mathrm{~b} / \mathrm{s} / \mathrm{Hz} / \mathrm{pol}$.) better performance than [6] for spectral efficiencies from 1 to $9 \mathrm{~b} / \mathrm{s} / \mathrm{Hz} / \mathrm{pol}$.

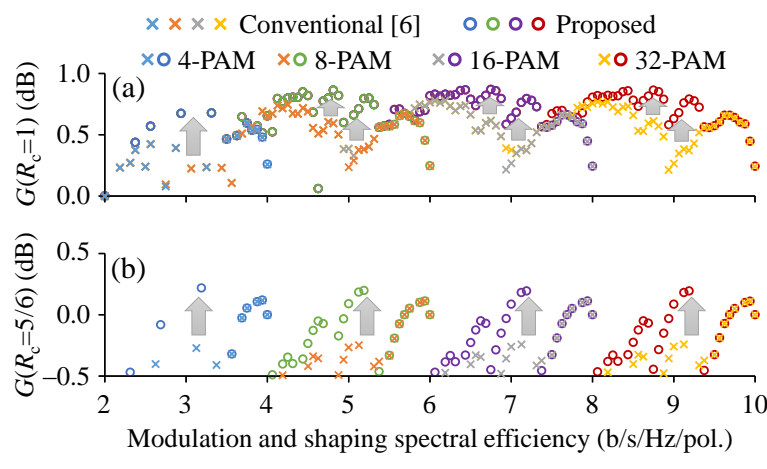

Fig. 5. Constellation gain as a function of modulation and shaping spectral efficiency by low-complexity DMs. Gray allows show improvement over [6].

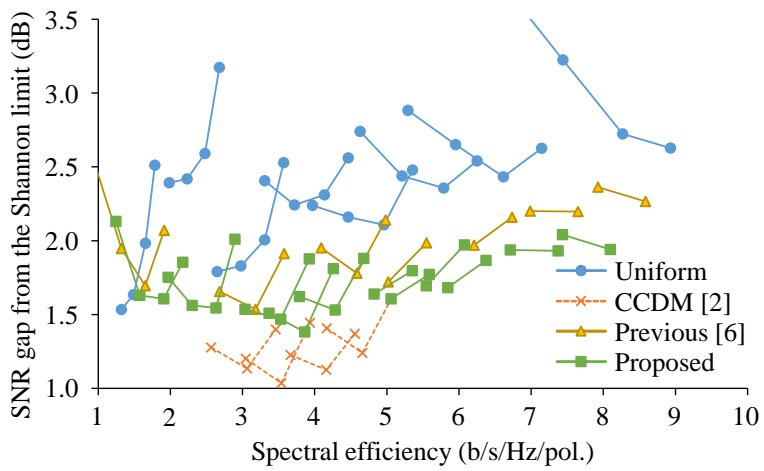

Fig. 6. SNR gap from the Shannon limit at the post-FEC BER of $10^{-15}$ with inner soft and outer hard-decision concatenation FEC codes.

\section{Conclusion}

We proposed a new bit-wise f2f-DM scheme with a variable-length output run-length mapper and distribution uniformalizers for probabilistically constellation shaping with significantly less complexity than arithmetic coding schemes. It does not require any multipliers. It is similar to stream processing, but is terminated periodically by the uniformalizer, thereby avoiding error propagation if the FEC decoder misconverges. It realizes at maximum $0.4 \mathrm{~dB}$ better performance (lower required SNR) than a previous low-complexity f2f-DM technique.

\section{References}

[1] G. Böcherer et al., "Bandwidth efficient and rate-matched lowdensity parity-check coded modulation," IEEE Trans. Commun., 63(12), p. 4651, 2015.

[2] P. Schulte et al., "Constant composition distribution matching," IEEE Trans. Inf. Theory, 62(1), p. 430, 2016.

[3] M. Yankov et al., "Constellation shaping for WDM systems using 256QAM/1024QAM with probabilistic optimization," J. Lightw. Technol., 34(22), p. 5146, 2016.

[4] M. Pikus and W. Xu, "Bit-level probabilistically shaped coded modulation," IEEE Commun. Lett., 21(9), p. 1929, 2017.

[5] G. Böcherer et al., "High throughput probabilistic shaping with product distribution matching," www.arxiv.org/pdf/1702.07510
[6] T. Yoshida et al., "Short-block-length by simple mark ratio controllers for granular and wide-range spectral efficiencies," in Proc. ECOC, 2017, p. Tu.2.D.2.

[7] T. V. Ramabadran, "A coding scheme for $m$-out-of- $n$ codes," IEEE Trans. Commun., 38(8), p. 1156, 1990.

[8] G. Böcherer et al., "Fast probabilistic shaping implementation for long-haul fiber-oiptic communication systems," Proc. ECOC, 2017, p. Tu.2.D.3.

[9] J. Cho et al., "Low-complexity shaping for enhanced nonlinearity tolerance," in Proc. ECOC, 2016, p. W1C.2.

[10]D. Subbotin, "Carryless rangecoder," Available:search.cpan.org

[11]ETSI, “Second generation ...," EN 302 307-1, v.1.4.1, 2014.

[12]D. Millar et al., "Detection of a $1 \mathrm{~Tb} / \mathrm{s}$ superchannel with a single coherent receiver," in Proc. ECOC, 2015, p. Mo.3.3.1. 